\title{
Effectiveness of an exercise programme on physical function in patients discharged from hospital following critical illness: a randomised controlled trial (the REVIVE trial)
}

\author{
Kathryn McDowell, ${ }^{1}$ Brenda O'Neill, ${ }^{1}$ Bronagh Blackwood, ${ }^{2}$ Chris Clarke, ${ }^{3}$ \\ Evie Gardner, ${ }^{4}$ Paul Johnston, ${ }^{5}$ Michaeline Kelly, ${ }^{6}$ John McCaffrey, ${ }^{7}$ Brian Mullan, ${ }^{8}$ \\ Sally Murphy, ${ }^{8}$ T John Trinder $^{9}{ }^{\text {Gavin Lavery, }}{ }^{8}$ Daniel F McAuley, ${ }^{2,4,8}$ Judy M Bradley ${ }^{2}$
}

- Additional material is published online only. To view please visit the journal online (http://dx.doi.org/10.1136/ thoraxjnl-2016-208723).

For numbered affiliations see end of article.

Correspondence to Dr Brenda O'Neill, Centre for Health and Rehabilitation Technologies (CHaRT), Institute of Nursing and Health Research, School of Health Sciences, Ulster University, Newtownabbey BT37 OQB, UK; b.oneill@ulster.ac.uk

For the trial protocol, see http:// trialsjournal.biomedcentral.com/ articles/10.1186/1745-621515-146

Received 5 April 2016 Revised 13 September 2016 Accepted 7 October 2016 Published Online First 15 November 2016

\section{SLinked}

- http://dx.doi.org/10.1136/ thoraxjnl-2016-209576

\section{ABSTRACT}

Objective To investigate the effectiveness of a 6 -week exercise programme in patients discharged home following critical illness compared with standard care. Design Multicentre prospective phase II randomised controlled trial, with blinded outcome assessment after hospital discharge, following the 6-week intervention and at 6 months.

Participants 60 patients (30 per group) aged $\geq 18$ years, mechanically ventilated $>96$ hours, and not in other rehabilitation, that is, cardiac or pulmonary rehabilitation programmes. Participants in the intervention group completed an individually tailored (personalised) exercise programme.

Outcome measures Primary outcome measure was SF-36 physical functioning following the intervention. Secondary outcomes included a range of performancebased and patient-reported measures.

Results Improvements in the primary outcome did not differ significantly between groups (mean difference ( $95 \% \mathrm{Cl}) 3.0(-2.2$ to 8.2$), p=0.26)$. The intervention group showed significant improvement compared with the control group (mean difference $(95 \% \mathrm{CI})$ ) in SF-36 role physical (6.6 (0.73 to 12.5$), p=0.03)$; incremental shuttle walk test ( $83.1 \mathrm{~m}(8.3$ to 157.9$), \mathrm{p}=0.03)$; functional limitations profile $(-4.8(-8.7$ to -0.9$)$, $\mathrm{p}=0.02)$; self-efficacy to exercise (2.2 (0.8 to 3.7$)$, $\mathrm{p}=0.01$ ) and readiness to exercise (1.3 (0.8 to 1.9 ), $\mathrm{p}<0.001)$. These improvements were not sustained at 6 months except readiness to exercise. Improvements in all other secondary outcome measures were not significant.

Conclusions There was no statistically significant difference in the primary outcome measure of selfreported physical function following this 6-week exercise programme. Secondary outcome results will help inform future studies.

Trial registration number NCT01463579. (results), https://dinicaltrials.gov/

\section{INTRODUCTION}

Globally, it is recognised that there is an urgent need to investigate interventions that could improve outcomes of patients after critical illness. It is estimated in the UK that approximately 100000 patients who are admitted to critical care annually survive to hospital discharge. These

\section{Key messages}

What is the key question?

- Does a 6-week personalised exercise programme improve physical function in patients discharged home from hospital following critical illness?

What is the bottom line?

- The exercise programme did not significantly improve physical function. Secondary outcome results will help inform future studies.

\section{Why read on?}

- To highlight the need for rehabilitation interventions to improve outcomes for patients following critical illness.

patients suffer from reduced physical function, exercise capacity, health-related quality of life and increased healthcare utilisation, which may continue for up to 5 years following discharge home from hospital. ${ }^{12}$

Studies investigating rehabilitation following critical illness have increased in recent years. However, there remains limited evidence to support rehabilitation following discharge from hospital for patients who survive critical illness as current studies show discordant results. ${ }^{3-11}$ Current guidelines in the $\mathrm{UK}^{12}$ highlight the lack of high-quality evidence to inform the appropriate timing and intervention for rehabilitation following critical illness. Further research is needed to inform the development of evidence-based guidelines for practice in line with other clinical populations where rehabilitation has been proven to be effective. ${ }^{13}$

The primary aim of this trial was to investigate the effectiveness of an individually tailored (personalised) 6-week exercise programme on physical function in patients discharged from hospital following critical illness compared with standard care. Second, we aimed to investigate the effectiveness of the 6-week programme of exercise on exercise capacity, health-related quality of life, anxiety and 
depression, and self-efficacy and readiness to exercise in patients discharged from hospital following critical illness compared with standard care; to determine the feasibility (safety, practicality and acceptability) of providing a 6-week programme of exercise for patients discharged from hospital following critical illness; and to explore the medium-term (6 months) effects of the exercise programme.

\section{METHODS}

\section{Design}

The study protocol has been described previously. ${ }^{14}$ In brief, this multicentre prospective phase II, allocation-concealed, assessor-blinded, randomised controlled clinical trial investigated the effectiveness of a 6-week personalised programme of exercise on patient outcomes following discharge from hospital after critical illness compared with standard care. The Northern Ireland Clinical Trials Unit (CTU) supported the conduct of the trial. The reporting of this trial adheres to the Consolidated Standards of Reporting Trials statement for randomised controlled trials (CONSORT) ${ }^{15}$ and the Template for Intervention Description and Replication (TIDieR). ${ }^{16}$

\section{Participants}

Participants were recruited from general intensive care units (ICUs) in six hospitals in Northern Ireland, UK. Eligible patients were aged $\geq 18$ years, had received mechanical ventilation for $>96$ hours, were planned to be discharged home, were medically fit to participate and were not participating in another rehabilitation programme, that is, cardiac rehabilitation or pulmonary rehabilitation. All patients gave informed consent to participate. Patients were randomly assigned to groups in a 1:1 ratio with the use of permuted blocks. Variable block sizes were used to ensure blinding. The randomisation schedule was generated using nQuery Advisor and allocations were done centrally online by the CTU which is located external to the study sites (allocation concealment).

\section{Intervention (exercise programme)}

The intervention group received standard care and completed a personalised exercise programme that consisted of two supervised and one unsupervised exercise session/s per week for 6 weeks. The exercise programme was planned to take 6 weeks to complete; however, it could be delivered over approximately 10 weeks to allow for participant non-attendance. Outpatient supervised sessions took take place in the hospital gymnasium, or if this was not possible, in the participant's home, and unsupervised sessions took place at home. The programme was delivered by a trained physiotherapist who worked closely with the critical care team. Physiotherapists' skills in exercise prescription, clinical reasoning and knowledge of the patient population facilitated the personalised nature of the programme. In addition, physiotherapists delivering the programme completed standardised training procedures and received a comprehensive intervention training pack including examples of how exercises could be personalised and progressed. The contents index of the training pack is included in the online supplementary material.

The exercise sessions consisted of (i) a warmup period; (ii) a circuit of $10 \mathrm{arm}$, leg and whole-body conditioning and strengthening exercises; (iii) an additional period of aerobic exercise (eg, walking, cycle ergometry or treadmill walking for at least $10 \mathrm{~min}$ and progressing as able up to a maximum of $30 \mathrm{~min}$ ) to maintain moderate breathlessness; and finally, (iv) a cool-down period and relaxation. The sessions lasted a maximum of 1 hour. Hand strengthening and dexterity exercises were also incorporated within the exercise sessions. Exercises were progressed to maintain a level of moderate breathlessness (3-4 Borg Breathlessness Scale). Strengthening exercises were included using higher repetitions and sets and an increase in weight for progression. The aerobic exercise was based, for example, on the patient's heart rate and/or the results of the incremental shuttle walk test (ISWT) measured at baseline assessment. Participants were provided with an exercise manual (available from http://www.science.ulster.ac.uk/inhr/revivemanual.pdf). The manual contained standardised descriptions and pictures of the exercises alongside space for the physiotherapist to document details of personalisation of the programme.

Protocols to manage patient safety were followed. There was a medical screening process to determine medical suitability of participants for the trial and then to review the status of each patient just prior to the baseline assessment to confirm safety to participate. The physiotherapist also determined the suitability of the participant to start and progress the exercises prior to and at their first appointment and at subsequent appointments through review of baseline information, medical notes, consultation with a designated research team member (and critical care consultant if needed) and clinical assessment. Modifications were implemented when indicated, for example, for an abdominal crunch if a patient was unable to adopt a supine position, the abdominal muscles were engaged by modifying this exercise to a seated position. Examples of such modifications, and condition-specific fact sheets and protocols, for example, for diabetes and cardiac conditions, were available to the physiotherapist as part of their intervention training pack.

Strategies to optimise treatment fidelity and ensure the intervention was delivered as intended were included, such as weekly phone calls with the research team to discuss individual patient treatment plans and regular training updates. ${ }^{17}$ Participants allocated to the intervention commenced the programme as soon as possible following baseline assessment. Participants allocated to the standard care group received no additional support after hospital discharge.

\section{Data collection and procedures}

Blinded outcome assessment was performed prior to randomisation at baseline (visit 1) (ideally within 2 weeks of hospital discharge or when the patient was deemed medically fit and/or able to attend), at visit 2 (following completion of the 6-week intervention) and at visit 3 (6 months following randomisation). Assessment was carried out by Northern Ireland Clinical Research Network research nurses independent of the research team and who were blinded to group allocation. Participants were instructed not to discuss their group allocation with the blinded outcome assessor.

\section{Outcome measures}

The primary outcome measure was physical function (change from baseline) as measured by the physical functioning (PF) subscale of the SF-36 Health Survey at visit 2 (following completion of the 6-week intervention or equivalent time points for the standard care group). ${ }^{18}$ Secondary outcome measures included a range of patient-reported and performance-based measures at 6 weeks and 6 months (table 1).

Feasibility as determined by safety, practicality and acceptability of the intervention was assessed by collecting data on the occurrence of adverse events, recruitment and retention, and the delivery and adherence to the exercise programme. Adherence was defined a priori as completion of $\geq 75 \%$ of supervised exercise sessions. At the beginning of the exercise 
Table 1 Secondary outcome measures

\begin{tabular}{|c|c|}
\hline & Outcome measures \\
\hline Physical function & Rivermead mobility index ${ }^{19}$ \\
\hline $\begin{array}{l}\text { Hand function (strength and } \\
\text { dexterity) }\end{array}$ & $\begin{array}{l}\text { Hydraulic hand dynamometer }{ }^{20} \\
\text { Nine hole peg test }{ }^{21}\end{array}$ \\
\hline Exercise capacity & Incremental shuttle walk test ${ }^{22}$ \\
\hline Health-related quality of life & $\begin{array}{l}\text { Remaining SF-36 Health Survey subscales and } \\
\text { component summary scores }{ }^{18} \\
\text { Functional limitations profile } \\
\text { EuroQol-5D-5L }\end{array}$ \\
\hline Breathlessness & Medical Research Council dyspnoea scale ${ }^{25}$ \\
\hline Anxiety and depression & Hospital anxiety and depression scale ${ }^{26}$ \\
\hline Readiness to exercise & Readiness to change questionnaire $\mathrm{e}^{27}$ \\
\hline Self-efficacy to exercise & $\begin{array}{l}\text { Chronic disease self-efficacy scale (exercise } \\
\text { component) }^{28}\end{array}$ \\
\hline
\end{tabular}

Healthcare utilisation questionnaire and semistructured interviews to assess cost effectiveness and patient views about the programme were also collected but not reported in this paper. These may be the focus of separate publications

programme, participants were asked to set a personal functional goal. After the programme, participants were asked to rate their confidence that they had achieved this on a numerical visual analogue scale (VAS).

\section{Statistical analysis}

There were limited data available in this specific research area at trial inception in order to conduct a formal sample size calculation. Without adequate meaningful data available, it is useful to use a more general recognised method, the Cohen method. ${ }^{29}$ Using this method, it was estimated that 52 patients (26 per group) would be required (Cohen's ${ }^{29}$ effect size of 0.5 ). On the basis of the previous experience of the research team, a loss of $25 \%$ after randomisation ${ }^{30}$ was anticipated and therefore we planned to recruit up to 68 participants (34 in each group), or until we achieved 52 (26 per group) completed data sets with the primary outcome measure at 6 weeks. Effectiveness of the intervention was analysed on an intention-to-treat basis. Standard approaches were used to detect patterns in missing data and imputation was performed using the group average. For the primary and other continuously distributed outcomes, differences between groups were tested using independent samples t-tests or non-parametric equivalents. Adjustments were made for baseline outcomes, baseline characteristics and other covariates as appropriate using analysis of covariance (ANCOVA). ANCOVA was also used to explore trends across time and differences between the groups. $\chi^{2}$ tests (or Fisher's exact tests) were used for categorical variables. The change from baseline was calculated by subtracting the baseline values from follow-up values, with between-group differences presented as means and 95\% CIs. Further information and exploratory subgroup analysis is detailed in the online supplementary material.

\section{RESULTS}

\section{Trial recruitment}

Recruitment occurred over a 3-year period from December 2011 until December 2014. Sixty participants (30 per group) were randomised. The participant flow through the trial is illustrated in figure 1. Participant baseline characteristics were not significantly different between study groups, except for gender (table 2). Baseline outcomes were significantly worse in the intervention group for SF-36 bodily pain and mental health $(\mathrm{MH})$ subscales, hospital anxiety and depression scale (HADS),
ISWT, functional limitations profile (FLP) psychosocial dimension and readiness to exercise (table 3 ). There was no significant difference between groups in the number of days following hospital discharge that participants attended visit 1 assessment (baseline) (table 2). Visit 2 (6-week follow-up) was completed by 55 participants ( 29 control group, 26 intervention group), and therefore, the predetermined sample size was achieved. Visit 3 (6-month follow-up) was completed by 49 participants (27 control group, 22 intervention group). There were no significant differences between groups in the time from visit 1 to visit 2 or from visit 1 to visit 3 (table 2).

\section{Delivery of the intervention}

The intervention was adhered to by 21 out of 30 participants (70\%) (ie, participant attended $\geq 75 \%$ of the intervention sessions according to the a priori definition of adherence). Reasons for non-adherence included unable to contact the participant $(n=3)$, exacerbation of asthma $(n=1)$, cancellations due to other commitments $(n=1)$ and no longer wishing to continue the intervention $(n=4)$.

The time taken to complete the intervention ranged from 6 to 11 weeks. Participants who took longer than 6 weeks to complete the exercise programme had cancellations due to general health issues such as feeling unwell, medical issues, family commitments or clinical appointments. One participant missed appointments due to relapse of alcohol dependence.

The intervention was designed to be group based over 6 weeks, but due to the recruitment rate and the distance between the trial sites the majority of supervised sessions were conducted individually. The majority of participants were willing to attend the hospital for all of their supervised exercise sessions. No participant required exclusively home visits for the exercise programme. Home visits were necessary in five participants on at least one occasion and otherwise all attended as outpatients. The main reasons for home visits in these participants were due to participants reporting they felt too acute to attend the hospital, travel distance too great to tolerate or unwilling to leave their house due to low mood.

At the end of the programme, participants rated their confidence (VAS 1-10) in achieving their preset personal functional goal as high (mean (SD), 9(1)). The majority (>80\%) of the planned components of the intervention were adhered to when cross-checked against the exercise case report form completed by the physiotherapist at each session, thus indicating a degree of high fidelity. ${ }^{17}$ Further information relating to the delivery of the intervention including key components of fidelity is given in the online supplementary material.

\section{Primary outcome: physical function}

For the primary outcome measure (SF-36 PF), improvement from visit 1 (baseline) to visit 2 (6 weeks) did not differ significantly between groups: mean difference 3.0 (95\% CI -2.2 to 8.2), $p=0.26$ (table 4). This difference in favour of the exercise group was equal to the minimal clinically important difference (MCID) reported for the general population. ${ }^{31}$ The primary outcome was missing for $8 \%$ of the participants. In order to adhere to the intention-to-treat principle, imputation (group average) was performed on the primary analysis but the result remained non-significant $(\mathrm{p}=0.30)$.

\section{Secondary outcomes}

The intervention group showed statistically significant improvement compared with the control group in the following secondary outcomes: SF-36 role physical (RP): mean difference 6.6 


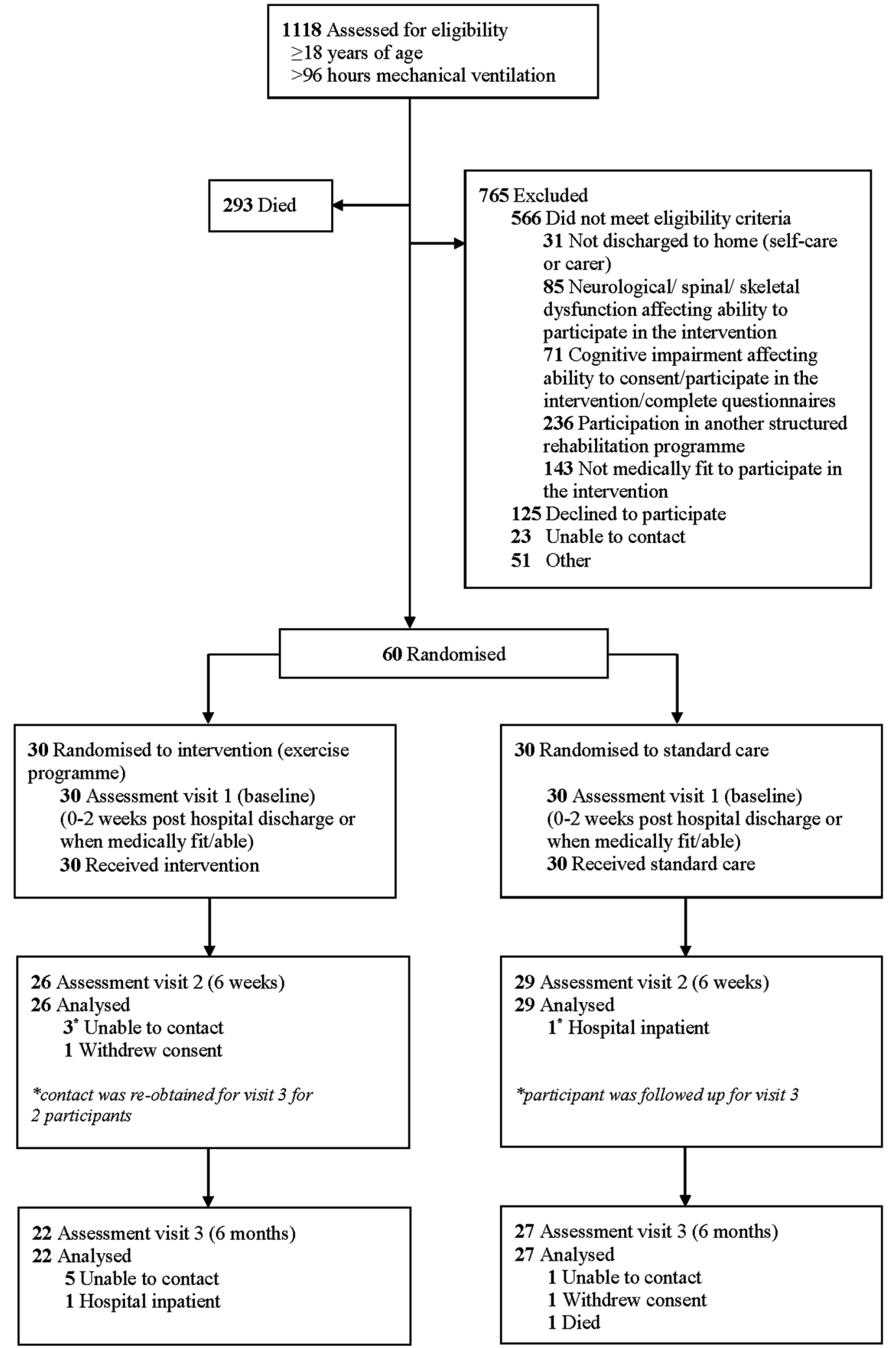

Figure 1 Screening, randomisation and follow-up of participants.

(95\% CI 0.73 to 12.5 ), $\mathrm{p}=0.03$; ISWT: mean difference $83.1 \mathrm{~m}$ (95\% CI 8.3 to 157.9 ), $\mathrm{p}=0.03$; FLP (overall score): mean difference -4.8 ( $95 \%$ CI -8.7 to -0.9$), p=0.02$; chronic disease self-efficacy scale (self-efficacy to exercise): mean difference 2.2 (95\% CI 0.8 to 3.7), $\mathrm{p}=0.01$; and readiness to change questionnaire (readiness to exercise): mean difference 1.3 (95\% CI 0.8 to 1.9), $\mathrm{p}<0.001$ (table 4). The difference in the improvement in SF-36 RP and ISWT in favour of the exercise group was also above the MCIDs reported in other populations. ${ }^{22}{ }^{31}$ Improvements in the remaining secondary outcome measures (remaining SF-36 subscales and component summary scores; Rivermead mobility index (RMI); EuroQol-5D-5L; hand-held 
Table 2 Participant baseline characteristics of intervention group and control group (visit 1)

\begin{tabular}{|c|c|c|}
\hline Variable & $\begin{array}{l}\text { Intervention } \\
(n=30)\end{array}$ & Control $(n=30)$ \\
\hline Age, years & $51(13)$ & $51(14)$ \\
\hline \multicolumn{3}{|l|}{ Gender } \\
\hline Male & 13 [43] & $21[70]$ \\
\hline Female & $17[57]$ & $9[30]$ \\
\hline \multicolumn{3}{|l|}{ ICU primary diagnosis } \\
\hline Respiratory & $17[56.7]$ & 13 [43.3] \\
\hline Cardiovascular & $4[13.3]$ & $4[13.3]$ \\
\hline Gastrointestinal & $3[10.0]$ & $6[20.0]$ \\
\hline Neurological & $2[6.7]$ & $3[10.0]$ \\
\hline Trauma & $2[6.7]$ & $3[10.0]$ \\
\hline Genitourinary & $1[3.3]$ & $0[0.0]$ \\
\hline Other & $1[3.3]$ & $1[3.3]$ \\
\hline APACHE 2 & $17.3(7.7)$ & $15.2(5.6)$ \\
\hline Length of stay in ICU, days & $\begin{array}{l}\text { Median (IQR) } \\
16.0(8.0-21.5)\end{array}$ & $\begin{array}{l}\text { Median (IQR) } \\
13.0(9.8-23.8)\end{array}$ \\
\hline Duration of mechanical ventilation, hours & $293.6(269.8)$ & $311.9(235.8)$ \\
\hline Length of stay in hospital, days & $\begin{array}{l}\text { Median (IQR) } \\
27.5(18.8-46.3)\end{array}$ & $\begin{array}{l}\text { Median (IQR) } \\
32.5(20.8-53.8)\end{array}$ \\
\hline Living alone & $5(16.7)$ & $7(23.3)$ \\
\hline $\begin{array}{l}\text { Time between hospital discharge and visit } \\
1 \text { (baseline), days }\end{array}$ & $48.9(29.4)$ & $44.3(28.6)$ \\
\hline $\begin{array}{l}\text { Time between visit } 1 \text { (baseline) and visit } \\
2 \text { (6 weeks), days }\end{array}$ & $78.19(26.6)$ & $73.3(21.0)$ \\
\hline $\begin{array}{l}\text { Time between visit } 1 \text { (baseline) and visit } \\
3 \text { ( } 6 \text { months), days }\end{array}$ & $191.8(26.6)$ & $183.1(20.6)$ \\
\hline
\end{tabular}

dynamometry; nine hole peg test; HADS; Medical Research Council (MRC) dyspnoea scale) did not differ significantly between groups (table 4). The difference in the improvement in the SF-36 physical component summary (PCS) and mental component summaries, and all SF-36 subscales apart from general health and $\mathrm{MH}$ in favour of the exercise group were above the MCIDs reported in the general population. ${ }^{31}$ Imputation analysis was also performed on secondary outcomes, which resulted in some additional secondary outcomes (SF-36 social functioning subscale (SF), SF-36 PCS and FLP physical dimension) moving from non-significant to significant. These results as well as additional FLP category scores are detailed in the online supplementary material.

For the ANCOVA, the following covariates were selected: SF-36 MH, HADS anxiety and depression, ISWT and FLP psychosocial dimension. These were selected due to differences at baseline that were deemed to be clinically important. In addition, it has been shown that patients with worse mental health report more physical limitations after adjustment for physical performance. ${ }^{32}$ Adjusted results remained statistically significant apart from FLP (overall score), and the FLP body care and movement category.

The primary analysis was repeated excluding the non-adherers in the intervention group (those attending $<75 \%$ of sessions). The overall trend for the results remained the same, but the improvement in the intervention group was larger. Further information is detailed in the online supplementary material.

Improvements in the outcome measures at visit 2 were not sustained at the 6-month follow-up assessment (visit 3) apart from readiness to exercise. This is described in more detail in figures 2-6.
Table 3 Participant baseline outcomes of intervention group and control group (visit 1)

\begin{tabular}{|c|c|c|}
\hline Variable & $\begin{array}{l}\text { Intervention } \\
(\mathrm{n}=30)\end{array}$ & $\begin{array}{l}\text { Control } \\
(n=30)\end{array}$ \\
\hline \multicolumn{3}{|l|}{ SF-36* } \\
\hline Physical functioning & $29.0(11.0)$ & $31.1(9.6)$ \\
\hline Role physical & $31.3(7.2)$ & $30.4(6.6)$ \\
\hline Bodily pain & $37.7(10.3)$ & $44.2(10.9)$ \\
\hline General health & $38.1(10.9)$ & $42.2(9.0)$ \\
\hline Vitality & $37.9(10.1)$ & $42.1(9.7)$ \\
\hline Social functioning & $29.7(13.5)$ & $33.9(13.4)$ \\
\hline Role emotional & $34.9(12.9)$ & $39.7(14.2)$ \\
\hline Mental health & $38.3(14.7)$ & $46.2(11.0)$ \\
\hline Physical component summary & $33.0(8.0)$ & $34.0(8.0)$ \\
\hline Mental component summary & $38.0(14.1)$ & $45.0(12.8)$ \\
\hline Rivermead mobility indext & $10.9(3.5)$ & $11.8(2.7)$ \\
\hline Incremental shuttle walk testł & $\begin{array}{l}n=28 \\
166.1(134.0)\end{array}$ & $\begin{array}{l}n=28 \\
258.6(171.7)\end{array}$ \\
\hline \multicolumn{3}{|l|}{ Functional limitations profile§ } \\
\hline Physical dimension & $28.0(16.7)$ & $21.2(15.8)$ \\
\hline Psychosocial dimension & $29.6(21.2)$ & $19.5(14.8)$ \\
\hline Overall score & $26.8(15.3)$ & $19.7(12.3)$ \\
\hline \multicolumn{3}{|l|}{ EuroQol-5D-5L } \\
\hline Visual analogue scaleף & $61.6(18.8)$ & $60.3(18.3)$ \\
\hline Index** & $0.5(0.3)$ & $0.6(0.2)$ \\
\hline Hand-held dynamometry†t & & $n=29$ \\
\hline Dominant hand & $60.4[31.2]$ & $66.9[20.1]$ \\
\hline Non-dominant hand & $58.5[34.7]$ & $67.4[21.9]$ \\
\hline Nine hole peg testł‡ & & $n=29$ \\
\hline Dominant hand & $75.9[16.1]$ & $79.0[13.4]$ \\
\hline Non-dominant hand & 77.2 [14.3] & $77.6[15.0]$ \\
\hline \multicolumn{3}{|l|}{ Hospital anxiety and depression scale§§ } \\
\hline Anxiety & $10.5(5.4)$ & $6.7(4.2)$ \\
\hline Depression & $7.6(4.9)$ & $5.1(3.3)$ \\
\hline Medical Research Council dyspnoea scaleףף & $\begin{array}{l}\text { Median (IQR) } \\
3.0(2.0-3.3)\end{array}$ & $\begin{array}{l}\text { Median (IQR) } \\
n=29 \\
2.0(1.5-3.0)\end{array}$ \\
\hline $\begin{array}{l}\text { Chronic disease self-efficacy scale (exercise } \\
\text { component)*** }\end{array}$ & $5.3[2.5]$ & $6.3[2.2]$ \\
\hline \multicolumn{3}{|l|}{ Readiness to exercisett† } \\
\hline Precontemplation & $1[3.3]$ & $0[0.0]$ \\
\hline Contemplation & $15[50.0]$ & $9[30.0]$ \\
\hline Preparation & $11[36.7]$ & $7[23.3]$ \\
\hline Action & $2[6.7]$ & $9[30.0]$ \\
\hline Maintenance & $1[3.3]$ & $5[16.7]$ \\
\hline
\end{tabular}

Values are mean (SD) or number [\%] unless stated.

${ }^{*}$ SF-36 scores are calculated from norm-based scores for a UK population with a mean of 50 and SD 10. A higher score represents better health-related quality of life (self-reported).

tRivermead mobility index range is $0-15$ with a higher score indicating better physical function (self-reported and performance based).

flncremental shuttle walk test range is $0-1020 \mathrm{~m}$ with a higher distance indicating better exercise capacity (performance based).

§Functional limitations profile range is $0-100$ with a lower score indicating better health-related quality of life (self-reported).

ๆVisual analogue scale range is 1-100 with a higher score indicating better health-related quality of life (self-reported).

**Index score is derived from value sets for a UK population with a lower score indicating better health-related quality of life (self-reported).

$\dagger+$ Percentage predicted score is calculated from norm-based UK values based on age and gender ${ }^{20}$ (performance based).

$\ddagger \ddagger$ Percentage predicated score is calculated from norm-based UK values based on age and gender ${ }^{21}$ (performance based).

$\S \S$ Hospital anxiety and depression scale range is $0-21$ with a higher score indicating a higher risk of anxiety/depression (self-reported).

११ीRange is $1-5$ with higher scores indicating greater disability due to breathlessness (self-reported).

${ }^{* * *}$ Range is $1-10$ with a higher score indicating better self-efficacy to exercise (self-reported).

t+tEach stage corresponds to the participants' motivational readiness to exercise (self-reported). 
Table 4 Outcome variables for intervention group and control group: mean (SD) change and mean difference (95\% Cl) from visit 1 (baseline) (visit 2 minus visit 1 )

\begin{tabular}{|c|c|c|c|c|}
\hline Outcome measure & $\begin{array}{l}\text { Intervention } \\
(n=26) \\
\text { Mean (SD) change }\end{array}$ & $\begin{array}{l}\text { Control } \\
(n=29) \\
\text { Mean (SD) change }\end{array}$ & $\begin{array}{l}\text { Difference mean } \\
\text { change scores } \\
(95 \% \mathrm{Cl})\end{array}$ & $\mathrm{p}$ Value \\
\hline \multicolumn{5}{|l|}{ SF-36* } \\
\hline Physical functioning & $6.8(10.9)$ & $3.9(8.2)$ & $3.0(-2.2$ to 8.2$)$ & 0.26 \\
\hline Role physical & $12.0(9.8)$ & $5.4(11.8)$ & $6.6(0.73$ to 12.5$)$ & 0.03 \\
\hline \multirow[t]{2}{*}{ Bodily pain } & $5.2(9.1)$ & $1.3(8.5)$ & $3.9(-0.87$ to 8.7$)$ & 0.11 \\
\hline & $\mathrm{N}=25$ & & & \\
\hline General health & $0.43(10.2)$ & $-1.2(7.8)$ & $1.7(-3.3$ to 6.6$)$ & 0.50 \\
\hline Vitality & $4.6(10.1)$ & $2.3(10.8)$ & $2.3(-3.4$ to 8.0$)$ & 0.42 \\
\hline \multirow[t]{2}{*}{ Social functioning } & $10.7(13.1)$ & $4.2(12.3)$ & $6.6(-0.3$ to 13.5$)$ & 0.06 \\
\hline & $\mathrm{N}=25$ & & & \\
\hline Role emotional & $8.2(14.5)$ & $2.5(16.4)$ & $5.7(-2.8$ to 14.2$)$ & 0.18 \\
\hline \multirow[t]{2}{*}{ Mental health } & $2.8(12.5)$ & $0.16(11.7)$ & $2.6(-3.9$ to 9.1$)$ & 0.43 \\
\hline & $N=25$ & & & \\
\hline \multirow[t]{2}{*}{ Physical component summary } & $7.0(7.8)$ & $3.2(6.7)$ & $3.8(-0.2$ to 7.8$)$ & 0.06 \\
\hline & $\mathrm{N}=25$ & & & \\
\hline \multirow[t]{2}{*}{ Mental component summary } & $5.8(13.6)$ & $1.1(13.1)$ & $4.6(-2.7$ to 11.9$)$ & 0.21 \\
\hline & $\mathrm{N}=22$ & $\mathrm{~N}=28$ & & \\
\hline \multirow[t]{2}{*}{ Rivermead mobility index* } & $1.3(2.1)$ & $1.1(1.8)$ & 0.13 (-0.98 to 1.2$)$ & 0.82 \\
\hline & $\mathrm{N}=20$ & $\mathrm{~N}=25$ & & \\
\hline Incremental shuttle walk test* & $135.5(119.8)$ & $52.4(126.7)$ & 83.1 (8.3 to 157.9 ) & 0.03 \\
\hline Functional limitations profilet & $\mathrm{N}=22$ & $\mathrm{~N}=28$ & & \\
\hline Physical dimension & $-10.0(9.7)$ & $-5.3(7.5)$ & $-4.7(-9.5$ to 0.2$)$ & 0.06 \\
\hline Psychosocial dimension & $-7.4(12.3)$ & $-2.4(9.2)$ & $-5.0(-11.1$ to 1.1$)$ & 0.11 \\
\hline Overall score & $-7.8(7.4)$ & $-3.0(6.3)$ & $-4.8(-8.7$ to -0.9$)$ & 0.02 \\
\hline EuroQol-5D-5L & $\mathrm{N}=22$ & $\mathrm{~N}=28$ & & \\
\hline Visual analogue scale* & $7.4(20.4)$ & $6.4(17.9)$ & $0.97(-9.9$ to 11.9$)$ & 0.86 \\
\hline Indext & $0.02(0.20)$ & $0.02(0.17)$ & $0.00(-0.1$ to 0.1$)$ & 0.998 \\
\hline Hand-held dynamometry* & $\mathrm{N}=22$ & $\mathrm{~N}=26$ & & \\
\hline Dominant hand & $6.1(28.1)$ & $12.5(22.8)$ & $-6.4(-21.2$ to 8.4$)$ & 0.39 \\
\hline Non-dominant hand & $9.1(28.2)$ & $14.0(28.7)$ & $-4.9(-21.5$ to 11.7$)$ & 0.56 \\
\hline Nine hole peg test* & $\mathrm{N}=22$ & $\mathrm{~N}=26$ & & \\
\hline \multirow[t]{2}{*}{ Dominant hand } & $9.4(15.3)$ & $5.3(10.7)$ & $4.1(-3.4$ to 11.7$)$ & 0.28 \\
\hline & $\mathrm{N}=21$ & $\mathrm{~N}=27$ & & \\
\hline Non-dominant hand & $8.9(13.7)$ & $5.3(16.1)$ & $3.6(-5.2$ to 12.4$)$ & 0.41 \\
\hline Hospital anxiety and depression scalet & $\mathrm{N}=22$ & $\mathrm{~N}=28$ & & 0.43 \\
\hline Anxiety & $-0.59(3.6)$ & $0.18(3.3)$ & $-0.77(-2.7$ to 1.2$)$ & 0.15 \\
\hline \multirow[t]{2}{*}{ Depression } & $-1.2(4.4)$ & $0.36(3.1)$ & $-1.6(-3.7$ to 0.6$)$ & \\
\hline & $\mathrm{N}=22$ & $\mathrm{~N}=27$ & & \\
\hline \multirow[t]{2}{*}{ Medical Research Council dyspnoea scale } & $-0.14(0.94)$ & $0.00(1.00)$ & $-0.14(-0.7$ to 0.4$)$ & 0.63 \\
\hline & $\mathrm{N}=22$ & $\mathrm{~N}=28$ & & \\
\hline \multirow[t]{2}{*}{ Chronic disease self-efficacy scale* } & $1.6(3.0)$ & $-0.57(2.1)$ & 2.2 (0.8 to 3.7 ) & 0.01 \\
\hline & $\mathrm{N}=22$ & $\mathrm{~N}=28$ & & \\
\hline Readiness to exercise* & $1.1(0.97)$ & $-0.21(0.96)$ & $1.3(0.8$ to 1.9$)$ & $<0.001$ \\
\hline
\end{tabular}

There was one unexpected serious adverse event that was assessed to be related to the intervention. This was a hospital admission following a recurrent acute exacerbation of asthma associated with anxiety, occurring at home within 24 hours of the intervention. Further details on adverse events are provided in the online supplementary material.

\section{DISCUSSION}

This study of a 6-week personalised exercise programme, initiated after discharge from hospital following critical illness, found no statistically significant difference in the primary outcome measure of self-reported physical function. The exercise programme resulted in statistically significant improvements in important patient-reported and performance-based secondary outcomes. These improvements were not sustained at 6 months except for readiness to exercise. Improvements in all other secondary outcome measures were not significant.

Patients with a longer length of stay in ICU and duration of mechanical ventilation than the majority of previous studies were included in this study. This was based on the hypothesis that these patients were more likely to have persisting disability and therefore would benefit more from a rehabilitation 


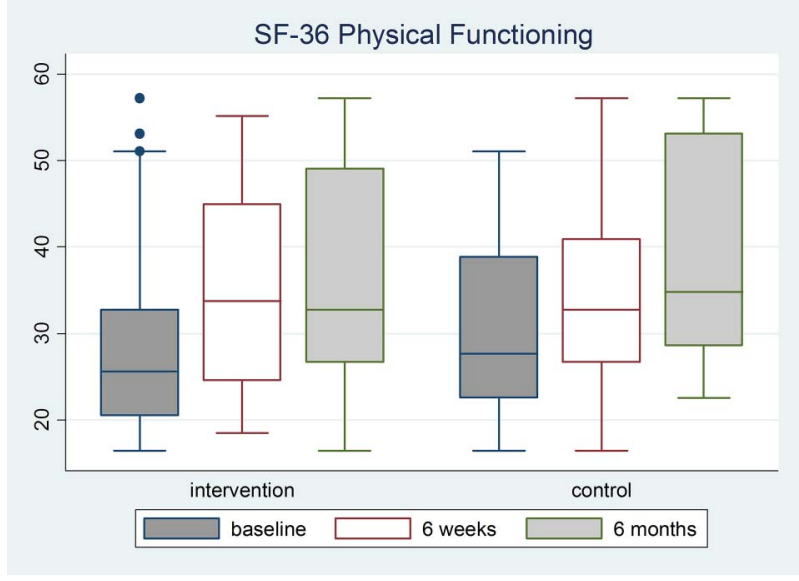

Figure 2 Trend across time for SF-36 physical functioning (PF) for each group (an increase represents improvement). The figure shows that there was an increase in PF across time in both groups; however, this increase was not statistically significant between groups $(0.79)$. The interaction between group and time was not significant $(p=0.08)$, indicating a similar pattern in both groups. There was no significant change in mean PF from baseline to 6 weeks $(p=0.26)$ and from baseline to 6 months $(p=0.79)$ between the two groups.

intervention. ${ }^{33}$ More robust approaches to identify patients for inclusion for rehabilitation are required, for example, those with higher degree of organ failure have also been associated with increased incidence and severity of ICU-acquired weakness. A recent study has identified that patients mechanically ventilated for $\geq 1$ week can be stratified into four disability risk groups based on age, ICU length of stay and functional dependency 7 days after ICU discharge. ${ }^{34}$ These groupings may be useful for future patient selection for rehabilitation. Due to the complex nature of this population, identification of patients for inclusion for rehabilitation is likely required to be based on individualised assessment taking into account these risk factors.

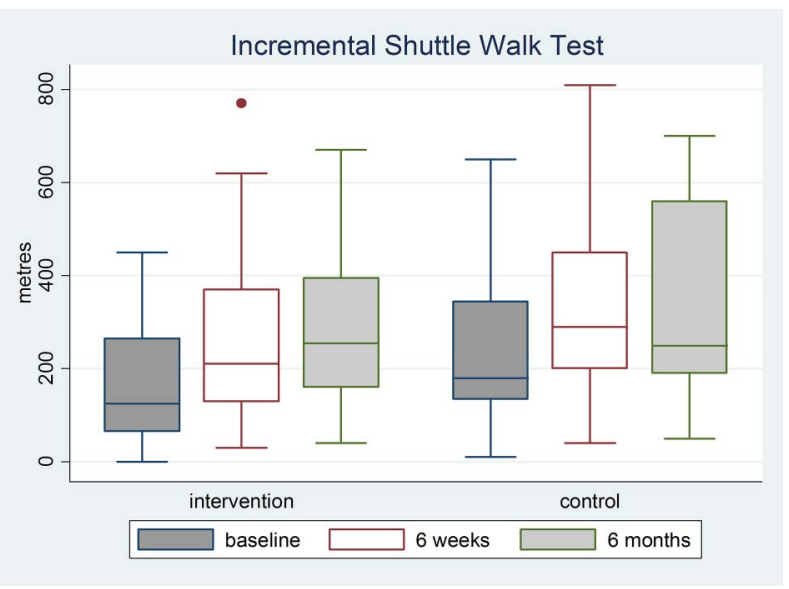

Figure 3 Trend across time for incremental shuttle walk test (ISWT) for each group (an increase represents improvement). The figure shows that there was an increase in ISWT across time in both groups; however, this increase was not statistically significant between groups $(p=0.33)$. The interaction between group and time was not significant $(p=0.13)$, indicating a similar pattern in both groups. There was a significantly $(p=0.03)$ larger increase in mean ISWT from baseline to 6 weeks in the intervention compared with the control group but no significant difference from baseline to 6 months $(p=0.16)$.

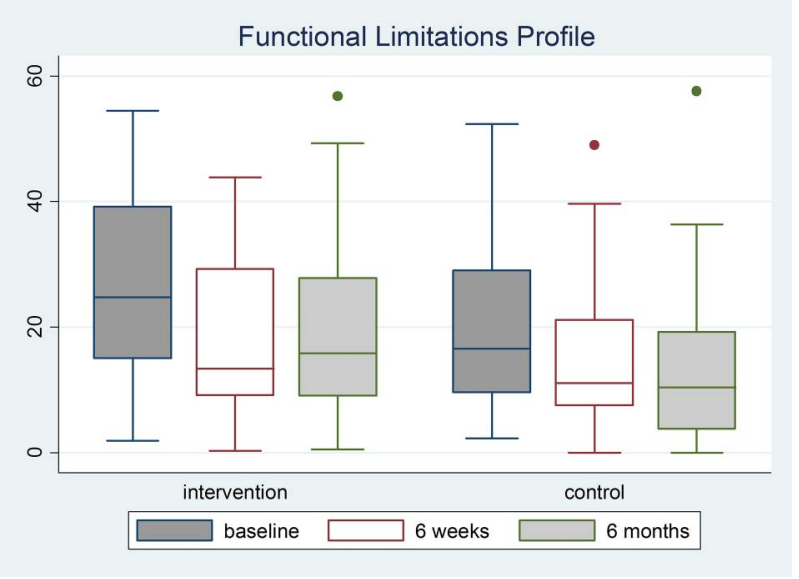

Figure 4 Trend across time for functional limitations profile (FLP overall score) for each group (a decrease represents improvement). The figure shows that there was a decrease in FLP across time in both groups; however, this decrease was not statistically significant between groups $(p=0.20)$. The interaction between group and time was significant $(p=0.01)$, indicating a different pattern in the two groups. There was a significantly $(p=0.02)$ larger decrease in mean FLP from baseline to 6 weeks in the intervention compared with the control group but no significant difference from baseline to 6 months $(p=0.81)$.

For our primary outcome of self-reported physical function (SF-36 PF), the difference was not statistically significant between groups. The SF-36 PF has demonstrated significant benefits of exercise rehabilitation in only one other comparable study. ${ }^{10}$ It is possible that the primary outcome measure selected might not have been sensitive enough to detect a difference in the intervention group, given that it is not a disease-specific measure. The SF-36 has gaps in areas that patients after critical illness have indicated are important. ${ }^{35}$ Development of critical care-specific outcome measures may be required and is the focus of a systematic review. ${ }^{36}$

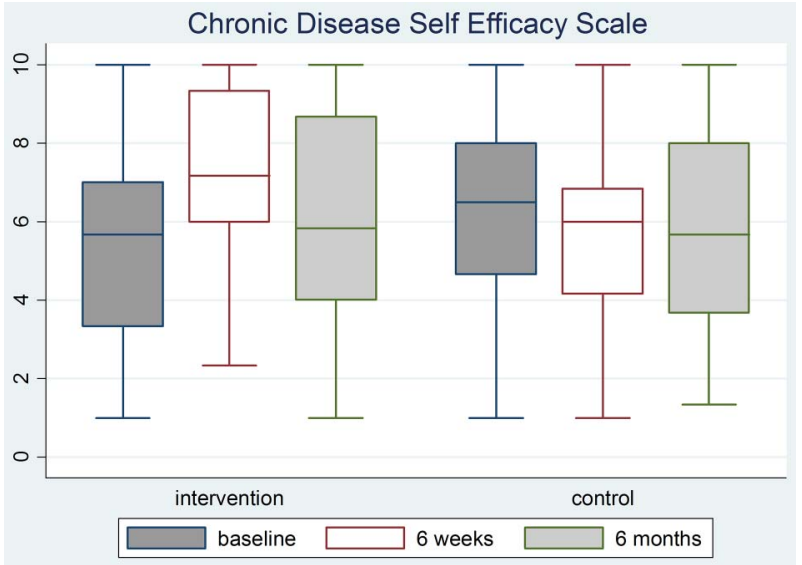

Figure 5 Trend across time for chronic disease self-efficacy scale (exercise component) for each group (an increase represents improvement). The figure shows that there was an increase in self-efficacy in the intervention group from baseline to 6 weeks but this was not maintained at 6 months. There was a decrease in self-efficacy across time in the control group. This resulted in a significant group xtime interaction $(p=0.04)$. There was a significantly $(p=0.01)$ larger increase in self-efficacy from baseline to 6 weeks in the intervention compared with the control group but no significant difference from baseline to 6 months $(p=0.08)$. 


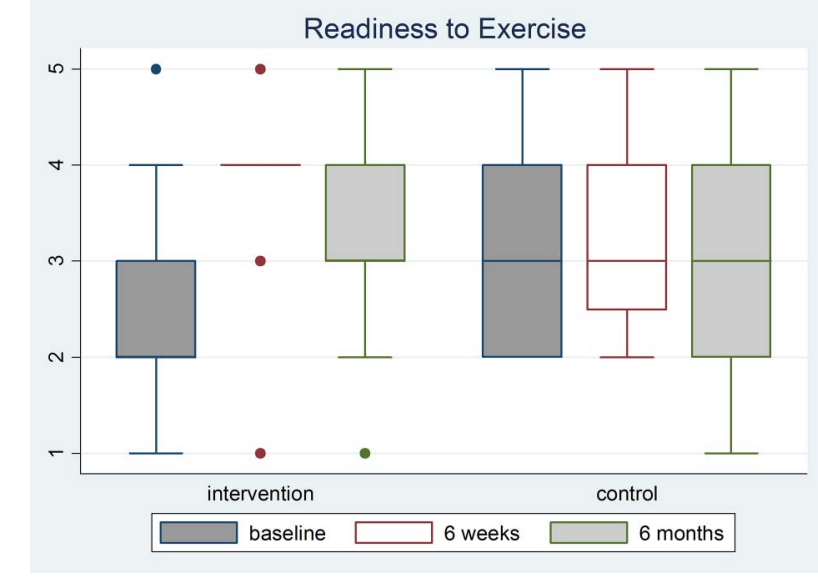

Figure 6 Trend across time for readiness to change questionnaire (readiness to exercise) for each group (an increase represents improvement). The figure shows that there was an increase in readiness to exercise across time in the intervention group but minimal change in the control group. This difference resulted in a significant group xtime interaction $(p=<0.001)$. There was a significantly $(p<0.001)$ larger increase in readiness to exercise from baseline to 6 weeks in the intervention compared with the control group, which was sustained at 6 months $(p=0.012)$.

In the absence of a consensus for the most appropriate outcomes to use in this population and critical care-specific outcomes, ${ }^{35} 36$ we included a range of self-reported and performance-based outcome measures as well as measures of self-efficacy and behaviour change relating to exercise in this population. The intervention group showed statistically significant improvements compared with the control group in some but not all secondary outcomes at 6 weeks, and therefore, it is difficult to draw strong conclusions from these results; however, findings may be useful in defining future studies. While some secondary outcome measure differences were above-published MCIDs, it is acknowledged that MCIDs for all outcomes were not available in the literature and none has been established specific to the postcritical care population. Validated field exercise tests such as the ISWT reflect functional exercise capacity and are recommended in other rehabilitation programmes. This study highlighted the benefit of using the ISWT as it demonstrated a clinically important and significant difference with the ISWT following the intervention, with an improvement of $83 \mathrm{~m}$. The FLP is a measure of health-related quality of life to evaluate individuals with varying types of and degrees of dysfunction. $^{23}$ The FLP demonstrated a statistically significant effect of the exercise programme in this study, whereas in contrast the $\mathrm{RMI}^{19}$ appeared to demonstrate a ceiling effect. This is the first study to assess and demonstrate significant improvements in self-efficacy and behaviour change relating to exercise in this population with the chronic disease self-efficacy scale (exercise component ${ }^{28}$ and readiness to change questionnaire (readiness to exercise) ${ }^{27}$ following the intervention. We wanted to explore participants' ability to change from not participating in exercise to exercising regularly as this represents a significant shift in their behaviour and may be important to include.

Our supervised exercise programme ended at 6 weeks, and the statistically significant improvements demonstrated in the outcome measures following the intervention were not sustained at 6 months. It has been reported that the training effect from exercise in healthy people declines at a rate of $1 \%$ a week once training ceases. ${ }^{37}$ Literature in other populations has similarly demonstrated the need for maintenance to sustain the benefits following short-term programmes. ${ }^{38}$ As part of this trial, we have explored our participants' perceptions of the programme of exercise and they indicated the need for support to continue exercise.

Our physiotherapy-led intervention included important elements previously shown to be effective for rehabilitation in other populations ${ }^{37-39}$ and was delivered with high fidelity. ${ }^{17}$ The intervention was standardised yet tailored to individual patient's needs facilitating exercise prescription and progression and the personal functional goals of participants. This is important for the heterogeneous postcritical care population.

The exercise programme in our trial was supervised twice per week by trained physiotherapists with knowledge of sequelae after critical illness. Other studies with supervised programmes have demonstrated some benefit, ${ }^{3} 57$ whereas a home-based study that did not incorporate a high level of supervision was unable to demonstrate improved outcomes. ${ }^{8}$ Supervision or support may be an important component of rehabilitation as it has been suggested that exercise can be difficult for people to perform without feedback about their performance or help to modify and progress the exercises. ${ }^{40}$

Our study has several limitations; only a small proportion of patients screened and survived to hospital discharge were recruited $(7.8 \%)$, which has implications for generalisability of the findings, although this is similar to the recruitment rate in the other rehabilitation studies following hospital discharge in this population. ${ }^{5}{ }^{8}$ Identification of which patients will benefit most from an exercise intervention based on baseline status has not yet been explored in the critical care population. Our analysis adjusted for the most clinically important baseline differences in order to minimise any impact on our results. We did not classify improvement based on level of ability at baseline, for example, ISWT distance, or explore the speed of recovery in our study due to small numbers. This could be an important outcome for future trials as exploratory analysis in a previous study showed that the rate of change over time (exercise capacity) was greater in the intervention group. ${ }^{6}$

A large proportion of patients were excluded due to attendance at other disease-specific rehabilitation programmes such as pulmonary or cardiac rehabilitation; these existing rehabilitation pathways were given priority over entry to the study as we anticipated that these patients may have disease-specific needs; for example, patients with COPD may need additional education and skills on inhaled therapies, which was not a feature of the REVIVE intervention but is a feature of pulmonary rehabilitation. Conversely, features of our rehabilitation may not be included in pulmonary rehabilitation. This highlights the need to explore the primary rationale for rehabilitation and whether the optimum rehabilitation would be the REVIVE intervention with additional components added.

Many patients were not medically fit to participate in the intervention or declined to participate in this study as they felt they had other health issues. It is possible that these patients were offered the intervention too early in their recovery when management of their other health issue was perceived as a priority, for example, delayed wound healing. Both physical and psychosocial health contribute to long-term disability, and the absence of psychosocial or educational components in our intervention may have reduced the effect. ${ }^{38}$ The small proportion of patients recruited and the distance between sites impacted on the plan for a group-based approach as the majority of sessions were completed individually. While a group bonding effect may have occurred if sessions were completed in a group format, 
conversely individualised delivery of the exercise intervention may be more appropriate for this heterogeneous population. The intervention ended at 6 weeks, and other than the short consultation at the final exercise session, there were no exercise maintenance strategies incorporated into the intervention. This may have contributed to the lack of sustained improvements at 6 months.

It is also recognised that our intervention targeted one phase of rehabilitation after critical illness while guidelines advocate seamless rehabilitation across the continuum of critical care recovery pathway. ${ }^{12}$ Nonetheless, research into interventions to support the immediate posthospital discharge period is required $^{11}$ and the results of our study support the need for a more flexible and personalised approach to commencing such an exercise programme. Targeting early modifiable risk factors, for example, depressive symptoms, may improve long-term outcomes of survivors of critical illness and may also optimise the rehabilitation programme. ${ }^{41}$ The cost effectiveness of our intervention has not yet been explored; however, costs for the delivery of other outpatient-based rehabilitation programmes have been estimated and shown to be cost effective. ${ }^{42}$

\section{CONCLUSIONS}

A 6-week personalised exercise programme, initiated after discharge from hospital following critical illness, found no statistically significant difference in the primary outcome measure of self-reported physical function. There were encouraging significant improvements in some, but not all secondary outcome measures that may be used to inform future studies. Improvements were not sustained at 6 months after the exercise intervention had been discontinued. A larger multicentre, phase III clinical trial of personalised exercise rehabilitation that also includes other components such as psychological support and behaviour change techniques and longer-term exercise maintenance strategies should be considered. Appropriate outcome measures for this population relevant to the goals of the rehabilitation should also be used in future trials.

\author{
Author affiliations \\ ${ }^{1}$ Centre for Health and Rehabilitation Technologies, Ulster University, \\ Newtownabbey, UK \\ ${ }^{2}$ Centre Experimental Medicine, School of Medicine, Dentistry \& Biomedical Sciences, \\ Queen's University Belfast, Northern Ireland, UK \\ ${ }^{3}$ Intensive Care Unit, Craigavon Area Hospital, Craigavon, UK \\ ${ }^{4}$ Northern Ireland Clinical Trials Unit, Belfast, UK \\ ${ }^{5}$ Intensive Care Unit, Antrim Area Hospital, Antrim, UK \\ ${ }^{6}$ Intensive Care Unit, Altnagelvin Area Hospital, Londonderry, UK \\ ${ }^{7}$ Intensive Care Unit, Belfast City Hospital, Belfast, UK \\ ${ }^{8}$ Regional Intensive Care Unit, Royal Victoria Hospital, Belfast, UK \\ ${ }^{9}$ Intensive Care Unit, Ulster Hospital, Dundonald, UK
}

Acknowledgements The authors wish to thank our patient representatives from the critical care patient liaison group (CritPaL) who provided public involvement and input to all aspects of the study. Also staff from the Northern Ireland Clinical Research Network (NICRN) who supported screening and recruitment and conducted blinded outcome assessment (Critical Care interest group), and provided support for the delivery of the exercise intervention (Respiratory Health interest group).

Contributors $K M C D$ and $B O^{\prime} N$ are joint first authors. DFMCA and JMB are joint senior authors. $\mathrm{KMCD}, \mathrm{BO} \mathrm{O}^{\prime} \mathrm{N}, \mathrm{BB}, \mathrm{DFMCA}$ and JMB contributed substantially to the study design, data acquisition, data analysis and interpretation, and the writing or revision of the manuscript. Original study concept was by DFMcA, SM, GL, JMB and BO'N. EG was the trial statistician. BO'N was chief investigator. BM was principal investigator (PI) at Royal Victoria Hospital; MK was PI at Altnagelvin Area Hospital; PJ was PI at Antrim Area Hospital; CC was PI at Craigavon Area Hospital; TJT was PI at Ulster Hospital; and JMcC was PI at Belfast City Hospital. All authors contributed to the revision of the manuscript and approved the final manuscript for submission.
Funding REVIVE, a charity of the Regional Intensive Care Unit, Northern Ireland. Additional funding provided by the Health and Social Care Research and Development Office, Northern Ireland. This trial was also supported by the Northern Ireland Clinical Research Network (NICRN) (Critical Care and Respiratory Health interest groups) and the Northern Ireland Clinical Trials Unit (NICTU), a UK Clinical Research Collaboration (UKCRC) registered clinical trials unit.

Competing interests None declared.

Patient consent Obtained.

Ethics approval The REVIVE trial was approved by the Northern Ireland Research Ethics Committee (ORECNI) (11/NI/0115).

Provenance and peer review Not commissioned; externally peer reviewed.

\section{REFERENCES}

1 Herridge MS, Tansey CM, Matté A, et al. Functional disability 5 years after acute respiratory distress syndrome. N Engl J Med 2011;364:1293-304.

2 Cuthbertson $\mathrm{BH}$, Roughton $\mathrm{S}$, Jenkinson $\mathrm{D}$, et al. Quality of life in the five years after intensive care: a cohort study. Crit Care 2010;14:R6.

3 McWilliams DJ, Benington SM, Atkinson D. Outpatient-based physical rehabilitation for survivors of prolonged critical illness: a randomized controlled trial. Physiother Theory Pract 2016;32:179-90.

4 Jones C, Eddleston J, McCairn A, et al. Improving rehabilitation after critical illness through outpatient physiotherapy classes and essential amino acid supplement: a randomized controlled trial. J Crit Care 2015;30:901-7.

5 Batterham AM, Bonner S, Wright J, et al. Effect of supervised aerobic exercise rehabilitation on physical fitness and quality-of-life in survivors of critical illness: an exploratory minimized controlled trial (PIX study). Br J Anaesth 2014;113:130-7

6 Denehy L, Skinner EH, Edbrooke L, et al. Exercise rehabilitation for patients with critical illness: a randomized controlled trial with 12 months follow up. Crit Care 2013:17:R156

7 Jackson JC, Ely EW, Morey MC, et al. Cognitive and physical rehabilitation of intensive care unit survivors: results of the RETURN randomized controlled pilot investigation. Crit Care Med 2012;40:1088-97.

8 Elliott D, McKinley S, Alison J, et al. Health-related quality of life and physical recovery after a critical illness: a multi-centre randomised controlled trial of a homebased physical rehabilitation programme. Crit Care 2011;15:R142.

9 Cuthbertson BH, Rattray J, Campbell MK, et al., PRaCTICaL study group. The PRaCTICaL study of nurse led, intensive care follow-up programmes for improving long term outcomes from critical illness: a pragmatic randomised controlled trial. BMJ 2009;339:b3723.

10 Jones $C$, Skirrow $P$, Griffiths RD, et al. Rehabilitation after critical illness: a randomized, controlled trial. Crit Care Med 2003:31:2456-61.

11 Connolly B, Salisbury L, O'Neill B, et al., for the ECACIP group. Exercise rehabilitation for recovery from critical illness following intensive care unit discharge. Cochrane Database Syst Rev 2015;(6):CD008632.

12 National Institute for Health and Care Excellence. Rehabilitation after critical illness. London, UK: NICE Clinical Guideline 83. http://www.nice.org.uk/nicemedia/live/ 12137/58250/58250.pdf

13 McCarthy B, Casey D, Devane $D$, et al. Pulmonary rehabilitation for chronic obstructive pulmonary disease. Cochrane Database Syst Rev 2015;2:CD003793.

14 O'Neill B, McDowell K, Bradley JM, et al. Effectiveness of a programme of exercise on physical function in survivors of critical illness following discharge from the ICU: study protocol for a randomised controlled trial (REVIVE). Trials 2014;15:146.

15 Schulz KF, Altman DG, Moher D, for the CONSORT Group. CONSORT 2010 Statement: updated guidelines for reporting parallel group randomised trials. BMJ 2010;340:с332.

16 Hoffmann TC, Glasziou PP, Boutron I, et al. Better reporting of interventions: template for intervention description and replication (TIDieR) checklist and guide. BMJ 2014;348:g1687.

17 Borrelli B. The assessment, monitoring, and enhancement of treatment fidelity in public health clinical trials. J Public Health Dent 2011;71(Suppl. 1):S52-63.

18 Ware JE, Snow KK, Kosinski M. SF-36 Version 2 health survey: manual and interpretation guide; Lincoln, RI: Quality Metric Inc, 2000.

19 Collen FM, Wade DT, Robb GF, et al. The rivermead mobility index: a further development of the rivermead motor assessment. Int Disabil Stud 1991;13:50-4.

20 Gilbertson L, Barber-Lomax S. Power and pinch grip strength recorded using the hand-held Jamar ${ }^{\circledR}$ dynamometer and $B+L$ hydraulic pinch gauge: British normative data for adults. Br J Occup Ther 1994:57:483-8.

21 Oxford Grice K, Vogel KA, Le V, et al. Adult norms for a commercially available Nine Hole Peg Test for finger dexterity. Am J Occup Ther 2003;57:570-3.

22 Singh SJ, Jones PW, Evans R, et al. Minimum clinically important improvement for the incremental shuttle walking test. Thorax 2008;63;775-7.

23 Patrick DL, Peach H, eds. Disablement in the community. Oxford, England: Oxford University Press, 1989. 
24 Herdman M, Gudex C, Lloyd A, et al. Development and preliminary testing of the new five-level version of EQ-5D (EQ-5D-5 L). Qual Life Res 2011;20:1727-36.

25 Bestall JC, Paul EA, Garrod R, et al. Usefulness of the Medical Research Council (MRC) dyspnoea scale as a measure of disability in patients with chronic obstructive pulmonary disease. Thorax 1999;54:581-6.

26 Zigmond AS, Snaith RP. The hospital anxiety and depression scale. Acta Psychiatr Scand 1983;67:361-70.

27 Marcus BH, Simkin LR. The stages of exercise behaviour. J Sports Med Phys Fitness 1993;33:83-8

28 Lorig K, Stewart A, Ritter $\mathrm{P}$, et al. Outcome measures for health education and other health care interventions. Thousand Oaks, CA: Sage Publications, 1996.

29 Cohen J. Quantitative methods in psychology. A power primer. Psychol Bull 1992:112:155-9.

30 O'Neill B, McKevitt A, Rafferty S, et al. A comparison of twice- versus once-weekly supervision during pulmonary rehabilitation in chronic obstructive pulmonary disease. Arch Phys Med Rehabil 2007;88:167-72.

31 Maruish ME, ed. User's manual for the SF-36v2@Health Survey. 3rd edn. Lincoln, RI: QualityMetric Inc, 2011.

32 Ruo B, Baker DW, Thompson JA, et al. Patients with worse mental health report more physical limitations after adjustment for physical performance. Psychosom Med 2008;70:417-21.

33 Jones C, Griffiths RD. Identifying post intensive care patients who may need physical rehabilitation. Clin Intensive Care 2011;11:35-8.

34 Herridge MS, Chu LM, Matte A, et al. The RECOVER program: disability risk groups \& 1-year outcome after $\geq 7$ days of mechanical ventilation. Am J Respir Crit Care Med 2016:194:831-44.
35 Lim WC, Black N, Lamping D, et al. Conceptualizing and measuring health-related quality of life in critical care. J Crit Care 2016;31:183-93.

36 Needham DM. Improving long-term outcomes research for acute respiratory failure. 2013. http://www.comet-initiative.org/studies/details/360?result=true

37 Garber CE, Blissmer B, Deschenes MR. American College of Sports Medicine Position Stand: quantity and quality of exercise for developing and maintaining cardiorespiratory, musculoskeletal and neuromotor fitness in apparently healthy adults: guidance for prescribing exercise. Med Sci Sports Exerc 2011:43: 1334-59.

38 Bolton CE, Bevan-Smith EF, Blakey JD, et al., British Thoracic Society Pulmonary Rehabilitation Guideline Development Group, on behalf of the British Thoracic Society Standards of Care Committee. British thoracic society guideline on pulmonary rehabilitation in adults. Thorax 2013;68(Suppl. 2):ii1-30.

39 Association of Chartered Physiotherapists in Cardiac Rehabilitation (ACPICR): Standards for physical activity and exercise in the cardiac population. http://acpicr. com/sites/default/files/Acpicr\%20standards_1.pdf

40 Latham NK, Harris BA, Bean JF, et al. Effect of a home-based exercise programme on functional recovery following rehabilitation after hip fracture. A randomised controlled trial. JAMA 2014;311:700-8.

41 Bienvenu OJ, Colantuoni E, Mendez-Tellez PA, et al. Depressive symptoms and impaired physical function after acute lung injury: a 2-year longitudinal study. Am J Respir Crit Care Med 2012;185:517-24.

42 National Institute for Health and Clinical Excellence. Chronic heart failure: management of chronic heart failure in adults in primary and secondary care. CG108. London: National Institute for Health and Clinical Excellence, 2010. http:/l www.nice.org.uk/guidance/index.jsp?action $=$ bylD\&0=13099. 